\title{
Exuberant pyoderma gangrenosum in a patient with autoimmune hepatitis*
}

\author{
Stephanie Galiza Dantas ${ }^{1}$ \\ Nurimar Conceição Fernandes ${ }^{1}$
}

Leonardo Pereira Quintella²

DOI: http:/ / dx.doi.org/10.1590/abd1806-4841.20174871

\begin{abstract}
Pyoderma gangrenosum is a rare neutrophilic dermatosis, which usually presents as ulcers with erythematous-violaceous undermined edges and a rough base with purulent or sanguinous exudate. It can be primary or associated with an underlying disease. However, rare cases of its association with autoimmune hepatitis have been described in the literature. Diagnosis is based on a characteristic clinical picture and ruling out other causes of ulcers. This paper aims to discuss the management of corticosteroid therapy and the importance of local treatment. We report a case with torpid evolution, presented with multiple and deep ulcers in a young patient with autoimmune hepatitis, causing pain and significant disability. We observed complete healing of lesions after two months of successful treatment.
\end{abstract}

Keywords: Autoimmune hepatitis; Hepatitis; Leg ulcer; Pyoderma; Pyoderma gangrenosum; Sodium carboxymethylcellulose; Skin Ulcer; Therapeutics; Ulcer

\section{INTRODUCTION}

Pyoderma gangrenosum (PG) is a rare neutrophilic dermatosis, with an estimated annual incidence of 3-10 cases per million in the population. ${ }^{1}$ It can present itself as a primary disorder or be associated with an underlying disease, particularly inflammatory bowel disease (IBD), arthritis, hematologic diseases, and malignant diseases. $^{2}$

Typical clinical presentation consists of a pustule or nodule that evolves into a painful ulcer with an undermined and well-defined erythematous-violaceous border. The base of the ulcer commonly has granulation tissue with purulent or sanguinous exudate. It is usually located on lower limbs and displays centrifugal growth. Other possible forms of presentation include vegetative, bullous, and pustular types.

Its frequent association with autoimmune diseases and pathergy corroborate the hypothesis of PG as an autoimmune disease. ${ }^{3}$ Pathergy, which occurs in about $30 \%$ of cases, consists of the appearance of new lesions or the worsening of pre-existing lesions after a local trauma. ${ }^{4}$

The diagnosis is made - in addition to its compatible clinical characteristics - by excluding other causes of similar-appearing cutaneous ulcerations - including infection, malignancy, and vasculitis - by histopathological examination and laboratory study.
The treatment of secondary PG should consider the treatment of the underlying disease. In primary PG, corticosteroid therapy is the gold standard, sometimes in combination with immunosuppressant therapy. ${ }^{5}$

This paper aims to discuss an exuberant case of PG in a young patient with autoimmune hepatitis (AIH), emphasizing corticosteroid therapy management and the importance of local treatment.

\section{CASE REPORT}

We report a 25-year-old female patient with recurrent ulcers on the lower limbs for the past four years. She reported onset with pustules, which progressed to ulceration in the left medial malleolus region. Later, she noticed other similar ulcers on both lower limbs, with disabling local pain. As a relevant medical record, she had type $1 \mathrm{AIH}$. She was on prednisone (20 mg daily) and azathioprine (100 mg daily) - precribed for AIH - and clobetasol cream $0.05 \%$.

Physical examination revealed a deep ulcerated lesion on the lateral side of the right lower limb (RLL), about $5 \mathrm{~cm}$ in diameter, reaching the subcutaneous tissue, with violaceous regular and undermined edges, and base with purulent exudate and granulation tissue. We also observed two other ulcerated lesions on the lateral side of the RLL and an ulcer on the medial side of the RLL with similar characteristics (Figure 1). Similar ulcers were observed in the

Received on 23.06.2015.

Approved by the Advisory Board and accepted for publication on 11.01.2016

* Work performed at Hospital Universitário Clementino Fraga Filho - Rio de Janeiro (RJ), Brazil.

Financial Support: None.

Conflict of Interest: None.

Department of Dermatology of the Universidade Federal do Rio de Janeiro (UFRJ) - Rio de Janeiro (RJ), Brazil.

Department of Pathology of the Fundação Oswaldo Cruz (Fiocruz) - Rio de Janeiro (RJ), Brazil.

C 2017 by Anais Brasileiros de Dermatologia 
anterior aspect of the left lower limb (LLL) and the medial aspect of the LLL - the latter particularly deep, with maceration on the edges and smaller adjacent ulceration. A total of seven ulcers were observed (Figure 2).
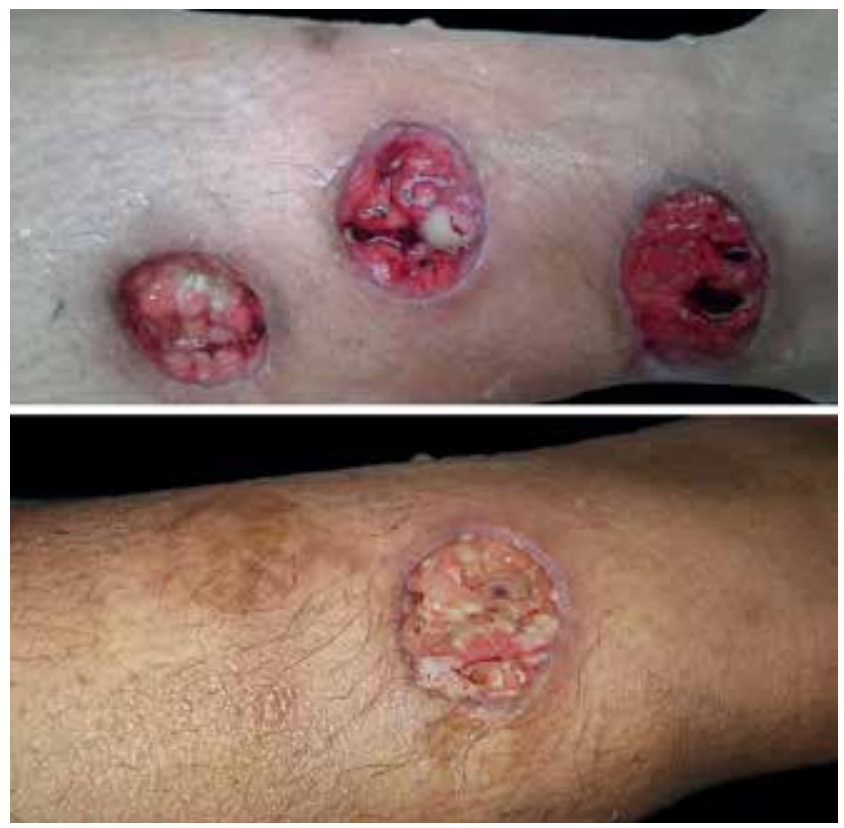

FiguRE 1: Right lower limb: aspect before treatment. Three deep ulcers on the lateral aspect and an ulcer in the medial region of the limb reaching the subcutaneous tissue, with violaceous regular and undermined edges
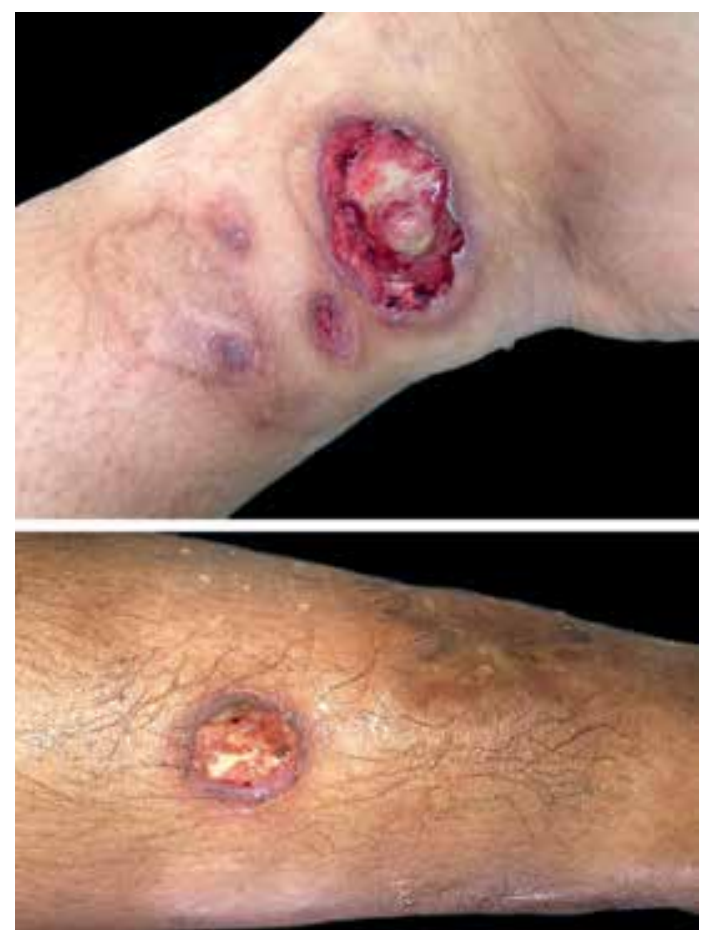

Figure 2: Left lower limb: aspect before treatment. On the left, deep ulcer with violaceous irregular and macerated edges in left medial malleolus region, with smaller adjacent ulceration. On the right, ulcer on the anteromedial aspect of the limb. We also observed atrophic scars caused by previous ulcers.
Histopathological study of a lesion revealed unspecific, ulcerated, and chronic inflammation process (Figures 3 and 4). The search for mycobacteria, fungi, and sporotrichosis serology were negative. PCR for leishmaniasis and leishmanin skin test were also negative. Laboratory tests revealed elevated C-reactive protein, glucose intolerance, and normal liver laboratory tests, except for the elevation of gamma-glutamyltransferase.

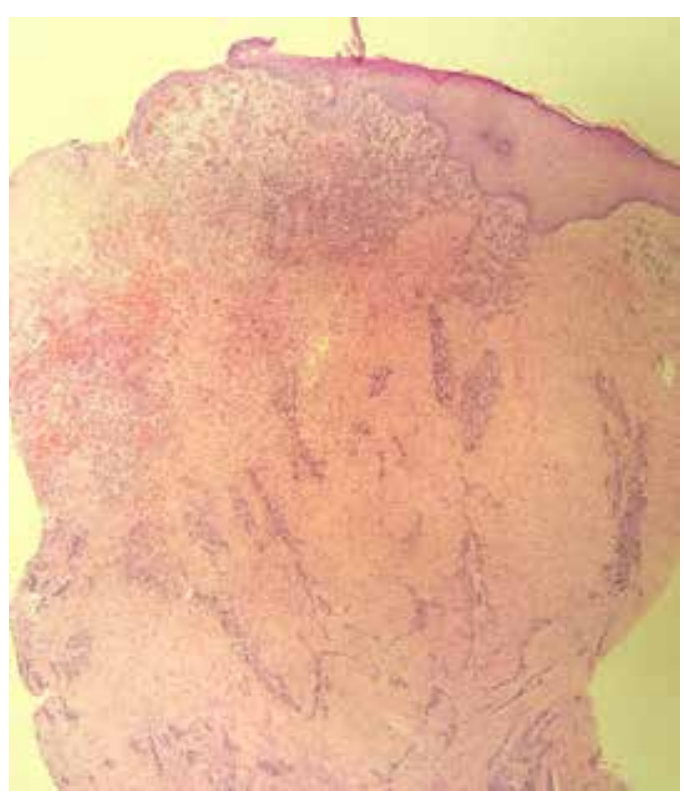

Figure 3: Histopathology. Ulcerated area with granulation tissue, recent hemorrhage, perivascular mononuclear inflammatory infiltrate and fibrosis (Hematoxylin \& eosin $x 40$ )

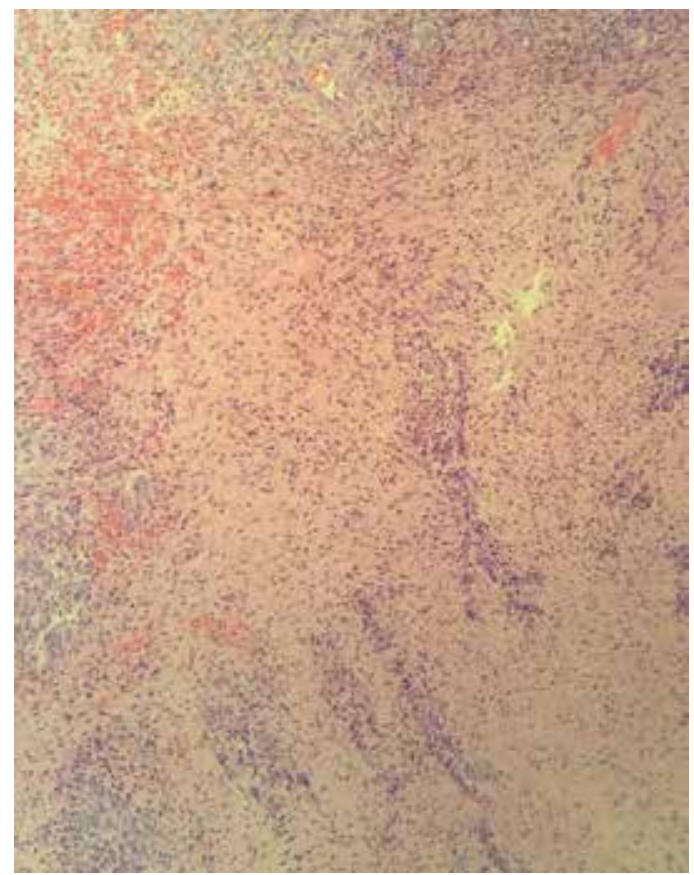

FIGURE 4: Histopathology. Histological detail showing inflammatory infiltrate composed of lymphocytes arranged around vessels (Hematoxylin \& eosin $x 100$ ) 

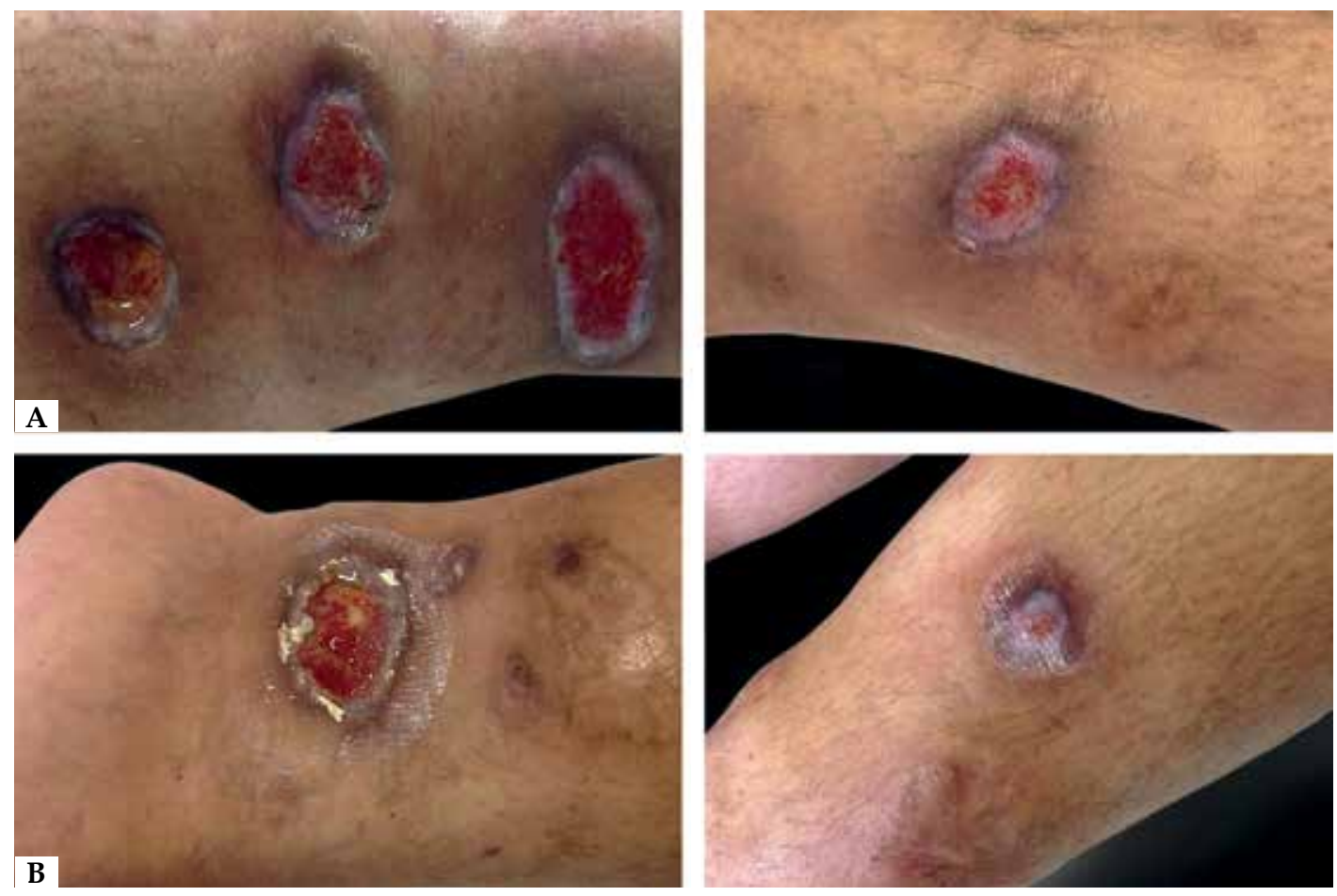

Figure 5:

One month after the initiation of treatment. Healing ulcers on the right (A) and left (B) lower limbs. Re-epithelialization occurs from the edges of the lesion

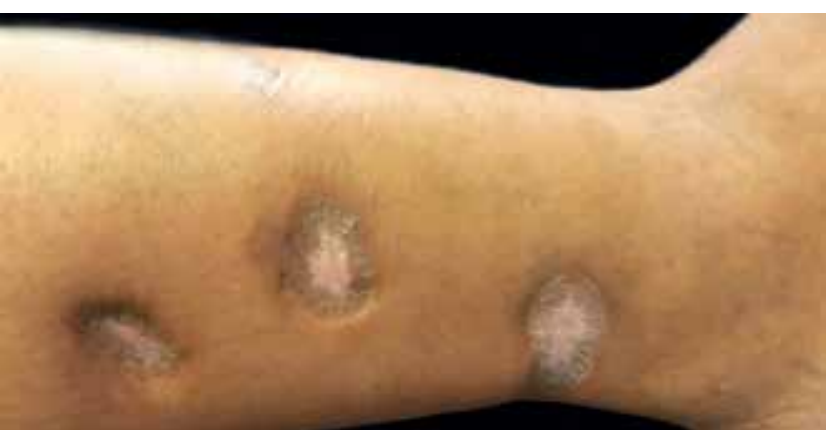

Figure 6: Three months after initiation of treatment. Complete healing of ulcers on the lateral aspect of the right lower limb

We started treatment with prednisone at a dose of $70 \mathrm{mg} /$ daily $(1 \mathrm{mg} / \mathrm{kg} /$ day). In the first week, we used dressings with silver sulfadiazine $1 \%$ on the lesions. From the second week, we used dressings with carboxymethyl cellulose $3 \%$ gel and Nystatin ointment with zinc oxide - the latter only on the macerated edge of the ulcer in the left medial malleolus region (candidiasis associated). We recommended resting and keeping the lower limbs elevated.

The patient was discharged after 18 days of hospitalization, with the prescription of prednisone $(60 \mathrm{mg} /$ daily) and daily dressings as described above, as well as guidance to rest and elevate the lower limbs. Initially, we programmed the reduction of Prednisone by $10 \mathrm{mg}$ per month. The patient returned a month after discharge showing healing process in the ulcers (Figure 5). Two months after discharge, the seven ulcers were completely resolved with use of prednisone $40 \mathrm{mg} /$ daily. Three months after discharge, we still observed the complete healing of ulcers with prednisone $30 \mathrm{mg} /$ daily and emollient. At the time, we reduced the prednisone dose to 20 $\mathrm{mg} /$ daily and kept emollient topical care (Figure 6).

\section{DISCUSSION}

Pyoderma gangrenosum (PG) is a rare dermatosis, and its association with autoimmune hepatitis (AIH) was rarely described in the literature. We report a case with torpid evolution, presenting with multiple and deep ulcers in a young patient with autoimmune hepatitis, causing pain and significant disability. Although the patient was treated with prednisone and azathioprine - both prescribed for $\mathrm{AIH}$ - the lesions showed no clinical improvement. In the present case, PG and the underlying disease did not follow parallel clinical courses, since AIH was inactive. It is known that the course of the PG can be parallel or not parallel in cases associated with underlying IBD. ${ }^{6}$

The systemic corticosteroid therapy is the treatment of choice for PG. High initial doses are often necessary, such as prednisone $60-80 \mathrm{mg} /$ daily. $^{7}$ In the present case, we initially prescribed prednisone $1 \mathrm{mg} / \mathrm{kg} /$ daily, an equivalent to $70 \mathrm{mg} /$ daily. There are reports on the successful use of azathioprine for PG treatment, but they still require further scientific evidence. ${ }^{8}$ We believe that the importance given to local treatment was essential for the healing of the lesions. In this particular case, we applied silver sulfadiazine topical $1 \%$, given the large amount of purulent exudate. Although it may be sterile in PG, we suspected secondary infection, considering the recent worsening of the lesion and the increased pain reported by the patient. Then we used carboxymethyl cellulose gel 3\%, one of the components of the hydrogel dressing, which has healing properties by aiding cell rehydration and autolytic debridement. ${ }^{9}$ Because of the high cost of commercial hydrogel dressing, we chose to use isolated carboxymethyl cellulose.

We further emphasize the slow withdrawal of corticosteroid therapy: after 17 days using $1 \mathrm{mg} / \mathrm{kg} /$ daily, given the evolutionary improvement observed, we started to reduce by $10 \mathrm{mg}$ every month; 
after $30 \mathrm{mg} /$ daily, we reduced $10 \mathrm{mg}$ every 15 days. We highlight the surprisingly satisfactory response to treatment, with complete healing of the lesions within two months. Currently, the patient is on prednisone $20 \mathrm{mg}$ / daily without remission. However, long-term follow up is necessary, given the variable course of PG. ${ }^{10}$

\section{REFERENCES}

1. Ahronowitz I, Harp J, Shinkai K. Etiology and management of pyoderma gangrenosum: a comprehensive review. Am J Clin Dermatol. 2012;13:191-211.

2. Konopka CL, Padulla GA, Ortiz MP, Beck AK, Bittencourt R, Dalcin DC. Pioderma Gangrenoso: um Artigo de Revisão. J Vasc Bras. 2013;12:25-33.

3. Coors EA, von den Driesch P. Pyoderma gangrenosum in a patient with autoimmunehaemolytic anaemia and complement deficiency. $\mathrm{Br} \mathrm{J}$ Dermatol. 2000;143:154-6.

4. Bonamigo RR, Razera F, Silveira G. Dermatoses neutrofílicas - Parte I. An Bras Dermatol. 2011;86:11-27.

5. Reichrath J, Bens G, Bonowitz A, Tilgen W. Treatment recommendations for pyoderma gangrenosum: an evidence-based review of the literature based on more than 350 patients. J Am Acad Dermatol. 2005;53:273-83.

6. Ruocco E, Sangiuliano S, Gravina AG, Miranda A, Nicoletti G. Pyoderma gangrenosum: an updated review. J Eur Acad Dermatol Venereol. 2009;23:100817.

7. Bolognia JL, Jorizzo JL, Rapini RP, editors. Pyoderma gangrenosum: Dermatology. London: Mosby; 2003. Vol.1. p.415-418.

8. Berth-Jones. Pyoderma gangrenosum. In: Treatament of Skin Disease comprehensive therapeutic strategies. Lebwohl MG, Heymann WR, Berth-Jopnes J, Coulson I, editors. 2nd ed. Lippincott Williams \& Wilkins: Philadelphia; 2006. p. 560-64.

9. Franco D, Gonçalves LF. Feridas cutâneas: a escolha do curativo adequado. Rev Col Bras Cir. 2008;35:203-6.

10. Binus AM, Qureshi AA, Li VW, Winterfield LS. Pyoderma gangrenosum: a retrospective review of patient characteristics, comorbidities, and therapy in 103 patients. Br J Dermatol. 2011 Dec;165:1244-50.

\author{
MAILING ADDRESS: \\ Stephanie Galiza Dantas \\ Rua Rodolpho Paulo Rocco, 255, $5^{\circ}$ andar \\ Secretaria de Dermatologia \\ Cidade Universitária \\ Ilha do Fundão \\ 21941-913 - Rio de Janeiro, RJ \\ Brazil \\ E-mail: stegaliza@gmail.com
}

How to cite this article: Dantas SG, Quintella LP, Fernandes NC. Exuberant pyoderma gangrenosum in a patient with autoimmune hepatitis. An Bras Dermatol. 2017;92(1):114-7. 